\section{E0061 THE MODULATION OF DILATED CARDIOMYOPATHY BY HEPC1 IN CTNTR141W TRANSGENIC MICE}

doi:10.1136/hrt.2010.208967.61

Zhang Li, Lv Dan, Dong Wei, Zhang Wei, Quan Xiongzhi, Wang Shumei. Comparative Medical Center Peking Union Medical College

Objective Iron regulatory hormone hepcidin is possible to be possibly involved in the physiological function of heart and pathogenesis of heart disease. The current paper reports the establishment of heart specific Hepc1 transgenic mice and analysis of the effect of Hepc1 on the development of dilated cardiomyopathy in the $\mathrm{cTnT}^{\mathrm{R} 141 \mathrm{~W}}$ transgenic mice.

Methods The heart specific Hepc1 transgenic mice were generated by the method of microinjection and the target gene was under the control of $\alpha$-MHC promoter. The expression level of the gene Hepc1 was determined by RT-PCR and slot blotting. The Hepc1 $1 \times \mathrm{cTnT}^{\mathrm{R} 141 \mathrm{~W}}$ double transgenic mice were generated by breeding the Hepc1 transgenic mice with $\mathrm{cTn}^{\mathrm{R} 141 \mathrm{~W}}$ transgenic mice. The structural and functional changes of the transgenic heart were analysed with M-mode echocardiography. Survival data were recorded from 1 month to 7 months (the $1^{\text {st }}$ to the $7^{\text {th }}$ month) postnatally. The histological and subcellular changes were observed under light microscope and transmission electronic microscope. The accumulation of phos-ERK1/2 and ERK1/2 in wild type, Hepc1, $\mathrm{cTnT}^{\mathrm{R} 141 \mathrm{~W}}$ and Hepc1 $1 \times \mathrm{cTnT}^{\mathrm{R} 141 \mathrm{~W}}$ transgenic mice were determined by western blotting. Phos-ERK1/2 expression was also detected in $\mathrm{H} 9 \mathrm{c} 2$ cell line, $\mathrm{H} 9 \mathrm{c} 2$ cell line treated with desferrioxamine (50 $\mu \mathrm{mol} / \mathrm{l})$, $\mathrm{H} 9 \mathrm{c} 2$ cell lines expressing $\mathrm{Hepc} 1$ protein and $\mathrm{H} 9 \mathrm{c} 2$ cell lines expressing Hepc1 protein treated with ferric ammonium citrate $(100 \mu \mathrm{mol} / \mathrm{l})$.

Results 2 heart specific Hepc1 transgenic mice lines were established. Hepc1 transgenic mice demonstrated no signs of cardiomyopathy or lethality up to 7 months of age. The immature death rate was $18 \%$ before 7 months of age in the $\mathrm{cTnT}^{\mathrm{R} 141 \mathrm{~W}}$ mice, while Hepc $1 \times \mathrm{cTnT}^{\mathrm{R} 141 \mathrm{~W}}$ transgenic mice showed a lower immature death $(10 \%)$. Hepc1 $\times c \operatorname{TnT}^{\mathrm{R} 141 \mathrm{~W}}$ transgenic mice showed a smaller ventricular chamber, improved left ventricular systolic function, and ameliorated interstitial fibrosis, compared with (to) $\mathrm{cTnT}^{\mathrm{R} 141 \mathrm{~W}}$ transgenic mice. ERK1/2 phosphorylation was strongly decreased in cTnT $^{\mathrm{R} 141 \mathrm{~W}}$ transgenic mice compared with (to) WT mice, while it was significantly increased in the Hepc $1 \times \mathrm{cTnT}^{\mathrm{R} 141 \mathrm{~W}}$ transgenic mice compared with (to) $c \operatorname{TnT}^{\mathrm{R} 141 \mathrm{~W}}$ transgenic mice. Both desferrioxamine, an iron chelator and the gene Hepc1 significantly increased ERK1/2 phosphorylation in $\mathrm{H} 9 \mathrm{c} 2$ cell line.

Conclusions Hepc1 showed a marked improvement on (in) the pathologic phenotype of DCM in cTnT ${ }^{\mathrm{R} 141 \mathrm{~W}}$ transgenic mice, in which ERK1/2 signal pathway may play an important role. Hepc1 may be a new target in the treatment of human dilated cardiomyopathy.

\section{e0062 THE RELATIONSHIP BETWEEN TWO POLYMORPHISMS IN CHRNA3 GENE AND NONSMALL CELL LUNG CANCER EVIDENCE FROM A METAANALYSIS}

doi:10.1136/hrt.2010.208967.62

\begin{abstract}
${ }^{1}$ Niu Wenquan, ${ }^{2}$ Gu Mingliang, ${ }^{3}$ Zhang Xuezhi, ${ }^{1}$ Guo Shujie, ${ }^{4}$ Qi Yue. ${ }^{1}$ Ruijin Hospital, Shanghai Jiaotong University School of Medicine, Shanghai, China; ${ }^{2}$ Chinese Academy of Sciences, Key Laboratory of Genome Science and Information Chinese Academy of Sciences, Chaoyang District, Beijing, China; ${ }^{3}$ Clinical Laboratory of Biochemistry, Central Hospital of Shengli Oil Field, China Petrochemical Corporation, Dongying, China; ${ }^{4}$ Capital Medical University, Affiliated Beijing Anzhen Hospital Beijing, Institute of Heart Lung \& Blood Vessel Diseases, Anzhenli, Beijing, China
\end{abstract}

Objective Lung cancer is the most common cause of cancer deaths worldwide; it is a complex disease that many genes involved (involves many genes) on chromosomes. Recently, several genome wide association studies have identified chromosomal region 15q2425.1 as a hotspot for lung cancer risk. We therefore aimed to explore the association of CHRNA3 gene rs1051730 and rs8034191 polymorphisms of this region with lung cancer via meta-analysis.

Methods Random-effects model was performed irrespective of the between-study heterogeneity. Data and study quality were assessed in duplicate. Publication bias was evaluated using the fail-safe number. Results Overall, five studies involving nine populations were identified for both rs1051730 (cases/controls: 7394/8784) and rs8034191 (9553/7953) polymorphisms. Genotype frequencies of two polymorphisms satisfied the Hardy-Weinberg law in controls. Presence of rs $1051730 \mathrm{~A}$ allele was significantly associated with 32\% increased risk of lung cancer ( $95 \%$ CI 1.25 to 1.39 ; $\mathrm{p}<0.00001$ ). Contrastingly, rs8034191 C allele only conferred a marginal association yielding $18 \%$ increased risk ( $95 \%$ CI 0.99 to $1.42 ; \mathrm{p}=0.07)$. Under assumption of dominant and recessive modes, risk magnitude was strengthened for rs 1051730 with an increased risk of $39 \%$ (95\% CI 1.30 to 1.48; $\mathrm{p}<0.00001)$ and $48 \%$ (95\% CI 1.25 to 1.76 ; $<<0.00001)$ for lung cancer, respectively. As for rs8034191, there was marginal or null association for the dominant (pooled $\mathrm{OR}=1.23 ; \mathrm{p}=0.06$ ) and recessive (pooled $\mathrm{OR}=1.26 ; \mathrm{p}=0.15$ ) modes. The fail-safe number at the level of 0.05 supported these significant associations.

Conclusions Our results demonstrated that the rs1051730 A allele was significantly associated with an increased lung cancer risk and the effect of rs8034191 polymorphism was moderate.

\section{E0063 CARDIOPROTECTION BY ISCHAEMIC PRECONDITIONING IS LOST IN ISOLATED DIABETIC HEARTS: ROLE OF TRANSIENT RECEPTOR POTENTIAL VANILLOID 1}

doi:10.1136/hrt.2010.208967.63

Song Jun-Xian, Chen Hong. Department of Cardiology, Peking University People's Hospital

Objective Extensive studies demonstrated that diabetes abolished the effectiveness of cardioprotection by ischaemic preconditioning (IPC) during ischaemia/reperfusion. Transient receptor potential vanilloid type 1 (TRPV1) contributes to the cardioprotective effect of IPC against ischaemia/reperfusion injury through the release of calcitonin gene-related peptide (CGRP) and substance P (SP). Our previous study found that the expression of TRPV1 receptor and the level of CGRP and SP were decreased in diabetic hearts. However, whether the underlying mechanism of the loss of cardioprotection by IPC during diabetes is associated with the impairment of TRPV1 receptor remains largely unknown.

Methods Isolated hearts from streptozotocin-induced diabetic rats and normal control rats were subjected to $30 \mathrm{~min}$ of global ischaemia followed by $40 \mathrm{~min}$ of reperfusion. IPC was initiated by 3 cycles of 5 min of global ischaemia and 5 min of reperfusion before prolonged ischaemia in the presence or absence of CGRP or SP receptor inhibitor Diabetic and normal control hearts were also pre-treated with CGRP or SP before prolonged ischaemia. Cardiac function parameters including left ventricular end-diastolic pressure, left ventricular developed pressure, maximum rise/fall rate of LV pressure, coronary flow and rate-pressure product and myocardial injury markers including creatine kinase $(\mathrm{CK})$ and cardiac troponin I (cTnI) in coronary effluent were monitored during the experiment. In addition, CGRP and SP release in coronary effluent during IPC were measured in the presence or absence of TRPV1 receptor inhibitors.

Results IPC effectively protected the hearts against ischaemia/ reperfusion injury by improving the recovery of cardiac function and lowering CK and cTnI release in coronary effluent in normal control rats, but not in diabetic rats. Pre-treatment with CGRP or SP significantly increased the recovery of cardiac function and decreased $\mathrm{CK}$ and cTnI release during ischaemia/reperfusion in both normal control and diabetic rats, and these cardioprotection of 
pre-treatment with CGRP or SP in normal control rats were comparable to those of IPC. In addition, inhibition of CGRP or SP receptor essentially abolished the cardiac protective effects of IPC in normal control rats, but not in diabetic rats. IPC resulted in significant increase of CGRP and SP release in coronary effluent of normal control hearts, and which were effectively inhibited by TRPV1 receptor inhibitor, capsazepine or ruthenium red. However, IPC had no effects on CGRP and SP release in coronary effluent of diabetic hearts in the presence or absence of capsazepine or ruthenium red. Conclusions Cardioprotection by IPC against ischaemia/reperfusion injury is lost during diabetes, and their underlying mechanism is partly associated with the decreased CGRP and SP release due to the impairment of TRPV1 receptor in diabetic hearts.

\section{e0064 MICRO RNAS ARE INVOLVED IN THE PSYCHOLOGICAL STRESS-INDUCED CARDIAC DISORDERS IN RATS}

doi:10.1136/hrt.2010.208967.64

Liu Bin, Wang Yuehui, Wang Lishuang, Zhao Zhuo, Wang Xiaodong. The Second Hospital of Jilin University

Objective Psychological stress has become an important factor in the development of cardiac disorders. MicroRNAs (miRNA) have been implicated in regulation of cardiovascular diseases. Therefore, we investigated whether miRNAs are involved in the psychological stress-induced cardiac disorders

Methods Stress rat models were established by complex stimulation at different times during the daytime for 2 weeks. Body weight, blood pressure and ECG were measured) every 3 days during processing stress. Adrenocorticotrophic hormone (ACTH) was measured by using ELISA. Cardiac changes were detected by HE staining and electronic microscopy. MicroRNA microarray was used for analysing the differential expression of miRNAs.

Results After psychological stress, rats displayed change in behaviour. Body weight increased slowly and systolic blood pressure increased significantly in stress group from the 6 th day to the 15 th day. The ECG of all rats was normal before experiment. 2 weeks after stimulation the ECG record of different individuals in stress group showed different arrhythmia, including sinus tachycardia, atrial premature contraction, ventricular arrhythmia, and ST-T changes. Hypothalamic-pituitaryadrenal activity increased in stress group compared to control group by detecting ACTH. And the ultrastructure and histology showed injury changes in stress group. Compared to the control group, there were 55 different miRNAs in stress model including upregulation of 20 and downregulation of left, among which miRNA-141, miRNA-382, miRNA-219-5p and miRNA-296 up-regulate, miRNA-135a and miRNA-466b are significant down-regulate.

Conclusions Complex stimulation can induce psychological stress, which can cause cardiac injury. MiRNAs change in stress rat models, including upregulation of miR-141, miR-382, miR-219-5p and miR296 , and significant downregulation of $\mathrm{miR}-135 \mathrm{a}$ and $\mathrm{miR}-466 \mathrm{~b}$, which may play important roles in psychological stress-induced cardiac disorders. (Supported by research grant NSFC 30940041).

\section{e0065 THE ASSOCIATION BETWEEN THE SINGLE NUCLEOTIDE POLYMORPHISMS OF MATRIX METALLOPROTEINASES AND THE CAROTID ATHEROSCLEROSIS IN PATIENTS WITH ESSENTIAL HYPERTENSION}

doi:10.1136/hrt.2010.208967.65

${ }^{1}$ Yan-Ping Ma, ${ }^{2}$ Xin-Juan Xu, ${ }^{2}$ Fang Zhao, ${ }^{2}$ Guang-Mei Hu, ${ }^{2}$ Jun-Shi Zhang. ${ }^{1}$ Department of Hypertension, The First Affiliated Hospital of Medical Univercity, Urumchi
Xinjiang, China; ${ }^{2}$ Department of Hypertension, The Xinjiang Armed Police Hospital, Urumchi Xinjiang, China

Objective To investigate the relationship between the single nucleotide polymorphisms (SNP) of matrix metallo proteases (MMP-2 -735C/T; MMP-3 -1171 5A/6A) and the carotid atherosclerosis (CAS) in Chinese Han and Uygur populations with EH.

Methods The study comprised 276 Han nationality and 212 Uygur participants, who were divided into two groups: CAS $(n=293)$ and NS $(n=195)$. Genotypes were detected by PCR-RFLP and their frequencies were determined.

Results (1) The frequencies of MMP-2 TT genotype and T allele in CAS were higher than in NS (Han : $X^{2}=11.441, p=0.003$; Uygur $\left.\mathrm{X}^{2}=28.255, \mathrm{p}=0.000\right)$. In NS, the frequencies of TT genotype and T allele in Han were higher than in Uygur $\left.\left(X^{2}=12.509, p=0.001\right)\right)$. (2) The frequencies of MMP-3 6A/6A genotype and 6A allele in CAS were higher than NS (Han: $X^{2}=7.523, p=0.024$; Uygur: $X^{2}=6.474$, $\mathrm{p}=0.039)$. The frequencies of $\mathrm{MMP}-36 \mathrm{~A} / 6 \mathrm{~A}$ genotype and $6 \mathrm{~A}$ allele in Han were higher than Uygur (CAS: $X^{2}=26.230, p=0.000$; NS: $\left.X^{2}=18.809, p=0.000\right)$. (3) The single gene analysis showed Han individuals with CT or TT genotypes had 2.25-fold risk and Han individuals with $6 \mathrm{~A} / 6 \mathrm{~A}$ genotypes had 1.85 -fold risk suffering from CAS. Han individuals with both T allele and $6 \mathrm{~A} / 6 \mathrm{~A}$ genotypes had 3.17-fold risk suffering from CAS. The single gene analysis showed that Uygur individuals with CT or TT genotypes had 5.04-fold risk suffering from CAS. Uygur individuals with 6A/6A genotypes had 2.20-fold risk suffering from CAS. Uygur individuals with both $\mathrm{T}$ allele and 6A/6A genotypes had 3.20-fold risk suffering from CAS. (4)According to MMP-2 genetypes, Han individuals with MMP-2 CT + TT genotypes had higher LDL and lower HDL levels than CC genotype in CAS (LDL:2.9 mmol/l vs $2.6 \mathrm{mmol} / \mathrm{l}$; HDL:1.2 mmol/1 vs $\mathrm{mmol} / \mathrm{l}$ ). Uygur individuals with CT + TT genotypes had higher TG levels than CC genotype (CAS : $2.5 \mathrm{mmol} / \mathrm{l}$ vs $1.6 \mathrm{mmol} / \mathrm{l}$; NS : $3.9 \mathrm{mmol} / \mathrm{l}$ vs $2.0 \mathrm{mmol} / \mathrm{l}$ ). According to $\mathrm{MMP}-3$, Han individuals with $6 \mathrm{~A} / 6 \mathrm{~A}$ genotype had higher T-CHOL and LDL levels than $5 \mathrm{~A} /$ $5 \mathrm{~A}+5 \mathrm{~A} / 6 \mathrm{~A}$ genotypes in NS group (T-CHOL : $4.6 \mathrm{mmol} / \mathrm{l}$ vs $4.2 \mathrm{mmol} / \mathrm{l}$; LDL: $2.3 \mathrm{mmol} / \mathrm{l}$ vs $2.2 \mathrm{mmol} / \mathrm{l}$ ). (5) The binary logistic regression analysis showed MMP-2 CT + TT genetypes were the risk factors for CAS in individuals with $\mathrm{EH}$ (Uygur: $\mathrm{OR}=9.65$; Han : $\mathrm{OR}=2.076$ ). MMP-3 6A homogeneses were the risk factors for CAS in Han individuals with $\mathrm{EH}(\mathrm{OR}=1.802)$. MMP-2 CT+TT and MMP-3 6A homogeneses had a combined influence on the incidence of CAS in Han individuals with $\mathrm{EH}$.

Conclusions (1) Han and Uygur individuals had differential distribution of MMPs. (2) The SNP of MMP-2 -735C/T is associated with CAS in individuals with EH. The MMP-2 T allele may be a risk factors on CAS in individuals with EH. The SNP of MMP-3 -1171 $5 \mathrm{~A} / 6 \mathrm{~A}$ is associated with CAS in Han individuals with $\mathrm{EH}$. The $6 \mathrm{~A}$ allele may be a risk factors on CAS in Han individuals with $\mathrm{EH}$.

\section{E0066 ISCHAEMIC PRECONDITIONING COMBINED WITH GRADUAL REPERFUSION OFFERS NO ADDITIONAL BENEFIT ON MITOCHONDRIAL PERMEABILITY PORE OVER PRECONDITIONING OR GRADUAL REPERFUSION ALONE}

doi:10.1136/hrt.2010.208967.66

Dong Chen, Lihuan Li. Fuwai Hospital

Objective Recent investigations demonstrate that ischaemic preconditioning and post-conditioning can reduce infarct size to the same degree. After reflow, opening of the mitochondrial permeability transition pore (mPTP) has been involved in lethal reperfusion injury. We hypothesised that the combination of ischaemic preconditioning and post-conditioning would result in greater 